\title{
Zur Aktualität der Psychologie des Scheiterns und Gelingens
}

\author{
Gedanken zur Neu-Erfindung einer Zeitschrift
}

\author{
Reinald Klockenbusch ${ }^{1}$
}

Online publiziert: 28. April 2016

(C) Springer Fachmedien Wiesbaden 2016

46 Jahre Zeitschrift „Gruppendynamik \& Organisationsberatung" werden markiert durch einen neuen Titel der Zeitschrift und eine Neu-Verortung des Themas als Teil der Organisationspsychologie, die ernst macht mit der Bedeutung von Gruppen und Interaktion in einem sich wandelnden Selbstverständnis im Spannungsfeld von Führung und partizipativer Gestaltung. Unsere Leser werden sich fragen: Was ist das Neue, was ist der Sinn dieses Unterfangens, und was hat es möglicherweise auch mit meiner eigenen Lebenswelt zu tun?

Als Verleger wurde ich von den Herausgeber/innen der Zeitschrift gefragt, warum mich dieses Thema selbst interessiert, warum ich mich für diesen Neu-Start engagiere, zusammen mit dem neuen Herausgeberteam. Was ist an dem Thema „Gruppe“ für mich als Verlags-Praktiker so spannend, dass ich mich selbst - zusammen mit den Herausgeber/innen - aktiv an der Neugestaltung des Themas beteiligt habe und mich als Teil des Erneuerungsprozesses verstehe?

Die Antwort ist, dass das Thema „Gruppe“ sehr viel mit dem zu tun hat, was die Bedingungen des Gelingens und des Scheiterns sind. Und diese Fragestellungen sind aus meiner Sicht aktueller denn je. Das folgende Beispiel möge dies umreißen.

In den Medien und in der Politik wurde in 2015 wohl kaum ein Satz so oft diskutiert wie der Satz „Wir schaffen das!“, das große Kanzlerinnen-Wort angesichts der Herausforderung einer großen Anzahl von Menschen, die es nach

Dr. Reinald Klockenbusch

reinald.klockenbusch@springer.com

1 Editorial Director Psychologie \& Springer Reference DE, Springer Fachmedien Wiesbaden GmbH, Abraham-Lincoln-Str. 46, 65189 Wiesbaden, Deutschland
Europa treibt, sogenannte Flüchtlinge, hinter denen Schicksale stehen, die hier nicht aufgezählt werden können. In den Medien wird der Satz „Wir schaffen das!“ manchmal bejubelt, manchmal verhöhnt. Wichtiger erscheint mir: Es ist ein Satz, der in jeder Organisation gesagt werden kann, bei der Change-Prozesse angesagt sind. Und es kommt sehr darauf an, wie glaubwürdig er geäußert wird. Wer ist mit dem „Wir“ gemeint? Ist es die Hybris vergangener Monarchen, der pluralis majestatis, der „Ich“ meint, wenn ,Wir“ gesagt wird? Ist es ein Appell an eine Gruppe von Mitarbeiter/ innen, sich zu fügen? Ist es die naive oder vereinnahmende Zuversicht, z. B. das berühmte „Pfeifen im Walde“? Oder ist es ein Satz, der sich nährt aus einer gelebten Führungskultur, bei der die Angesprochenen in die Lösung gemeinsamer Aufgabenstellungen einbezogen und ernstgenommen werden?

Fatal ist der Blick auf den/die einsame/n Entscheider/in unter Ausblendung der Kontexte. Dies ist eine der Ursachen von Fehl-Entscheidungen und Manipulationen. Letztlich lernen wir in Organisationen angesichts der Herausforderungen, die in Begegnungen sichtbar werden. Wodurch deutlich wird: Jede Begegnung ist eine Chance, wenn sie als solche wahrgenommen wird, wenn sich der Blick erweitert, um angemessenere Entscheidungen zu fällen.

Zum Satz der Kanzlerin ,Wir schaffen das!“": Ein vordergründiges Verständnis sagt nur etwas aus über das Gelingen. Wichtiger ist aber die Frage: Was sind die Bedingungen der Möglichkeit des Gelingens angesichts der Gefahr des möglichen Scheiterns? Und was bedeuten diese Bedingungen angesichts der Akteure, die etwas zum Gelingen beitragen können? Sofern sich diese Fragen auf Organisationen allgemein beziehen, sind sie Gegenstand der vorliegenden Zeitschrift. Und sie sind daher besonders aktuell, da es kaum eine Branche gibt, die sich nicht Veränderungsnotwendigkeiten stellen muss. Stichworte wie „Disruption“ 
oder „Industrie 4.0“ sind nur einige Beispiele, die die Randbedingungen kennzeichnen, warum sich heute Organisationen stets neu erfinden müssen.

Und dies gilt eben auch für das Thema der Gruppendynamik und die vorliegende neue Zeitschrift. Die Herausgeber/innen und der Verlag möchten sich Ihnen, liebe Leser/ innen stellen. Den Praktiker/innen und den Wissenschaftler/ innen, den Autor/innen. Und Sie beteiligen, damit wir dem Thema der partizipativen Führung und der Bedeutung von Gruppenprozessen in Organisationen auch hier ein Stück gerechter werden; um vielleicht nicht gleich fertige Antworten zu geben, aber um mit Ihnen in die Möglichkeit der Beantwortung von Fragen hineinzuleben.

Konkret freuen wir uns über kritisches und konstruktives Feedback, z. B. an iwers-stelljes@gio.de oder auch an mich im Verlag via reinald.klockenbusch@springer.com. Darüber hinaus möchten wir Ihnen die Möglichkeit geben, sich über die Veränderungsprozesse hinsichtlich unserer Zeitschrift selbst zu informieren und sich an den Diskussionen und Innovationsprozessen zu beteiligen, damit wir auch diese Zeitschrift in Zukunft noch besser machen können. Bitte merken Sie sich vor: Autor/innen und Leser/innen werden eingeladen zum ,Journal Day Springer Psychologie 2016“, der am 4. November 2016 in Berlin stattfinden wird von 11-19 Uhr. Anmeldungen an elke.janosch@springer.com bis zum 15.07.2016.

Der Verlag dankt den Herausgeber/innen und Leser/ innen und wünscht zum Neustart der Zeitschrift und allen weiteren Schritten gutes Gelingen.

Dr. Reinald Klockenbusch Jahrgang 1957, ist Editorial Director für die Bereiche Psychologie und ReferenceWorks im Springer-Verlag. Studium der Philosophie, Physik und Geschichte der Naturwissenschaften in Mainz mit Abschlüssen Lehramt Gynmasium und Promotion. Dissertations-Preis und Lehraufträge der Universität Mainz. Berufliche Weiterbildungen in Organisationsentwicklung (TOPs Berlin) und Coaching (Gisela Knopp). 